\title{
The effect of different number of diffusion gradients on SNR of diffusion tensor-derived measurement maps
}

\author{
Na Zhang ${ }^{1}$, Zhen-Sheng Deng ${ }^{1 *}$, Fang Wang ${ }^{1}$, Xiao-Yi Wang ${ }^{2}$ \\ ${ }^{1}$ Institute of Biomedical Engineering, School of Info-physics and Geomatics Engineering, Central South University, Changsha, Hunan, P.R. China \\ (410083); ${ }^{2}$ Department of Radiology, XiangYa Hospital of School of Medicine, Central South University, Changsha, Hunan, P.R. China \\ (410008); ${ }^{*}$ Corresponding author: Zhensheng Deng (bmedzs@csu.edu.cn or dengzhensheng@hotmail.com)

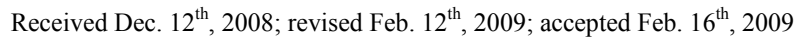

\begin{abstract}
Diffusion tensor imaging (DTI) is mainly applied to white matter fiber tracking in human brain, but there is still a debate on how many diffusion gradient directions should be used to get the best results. In this paper, the performance of 7 protocols corresponding to $6,9,12,15,20,25$, and 30 noncollinear number of diffusion gradient directions (NDGD) were discussed by comparing signal-noise ratio (SNR) of tensor- derived measurement maps and fractional anisotropy (FA) values.
\end{abstract}

All DTI data (eight healthy volunteers) were downloaded from the website of Johns Hopkins Medical Institute Laboratory of Brain Anatomical MRI with permission. FA, apparent diffusion constant mean (ADC-mean), the largest eigenvalue (LEV), and eigenvector orientation (EVO) maps associated with LEV of all subjects were calculated derived from tensor in the 7 protocols via DTI Studio. A method to estimate the variance was presented to calculate SNR of these tensor-derived maps. Mean \pm standard deviation of the SNR and FA values within region of interest (ROI) selected in the white matter were compared among the 7 protocols.

The SNR were improved significantly with NDGD increasing from 6 to $20(P<0.05)$. From 20 to 30, SNR were improved significantly for LEV and EVO maps $(P<0.05)$, but no significant differences for FA and ADC-mean maps ( $P>0.05)$. There were no significant variances in FA values within ROI between any two protocols ( $P>$ 0.05).

The SNR could be improved with NDGD increasing, but an optimum protocol is needed because of clinical limitations.

Keywords: Diffusion Tensor Imaging, Diffusion Gradient, Signal Noise Ratio, Estimating Variance

\section{INTRODUCTION}

Diffusion tensor imaging (DTI) has emerged as a noninvasive magnetic resonance imaging (MRI) modality capable of providing in vivo fundamental information of the white matter structure, which is required for viewing structural connectivity in the human brain $[1,2]$. It is commonly used to demonstrate subtle abnormalities in a variety of diseases (including stroke, multiple sclerosis, dyslexia, and schizophrenia) and is currently becoming part of many routine clinical protocols [3]. The principle of DTI is based on diffusion anisotropy of water molecular. By acquiring diffusion weighted (DW) images with diffusion gradients oriented in at least six noncollinear directions (The tensor has 6 independent parameters, that is why the minimal number of diffusion gradient directions (NDGD) for DTI measurement is 6), it is possible to measure the diffusion tensor modeled by a 3 dimension (3D) ellipsoid in each voxel [4,5]. The tensor-derived matrices, like diffusion anisotropy maps and color-coded orientation maps, which could characterize specific features of the diffusion process, can be calculated from tensor via DTI Studio [6]. DTI always operates under the assumption of a single ellipsoid. For estimation of more complex geometries, high angular resolution diffusion imaging (HARDI) or Q-Ball Imaging needs to be used [7].

NDGD is one of the most important factors for DW images acquisition. As NDGD increasing, more DW images are used to calculate the diffusion tensor, resulting in more accurate tensor estimation but much longer imaging time. Considering signal-noise ratio (SNR), if the same amount of time is used to acquire DW images, one 6-diffusion gradients is used, and the other 12-diffusion gradients, in the former, more images are acquired in each direction, which results in more averages, but in the latter, less averages. So which is better for single 3D ellipsoid estimation is still open to debate.

Some researchers $[8,9]$ claimed that more than 6 diffusion gradients can provide better measurements of the tensor than the conventional 6-diffusion gradients. A 
recent study with Monte Carlo simulations[10] concluded that at least 20 NDGD were necessary for a robust estimation of diffusion anisotropy, whereas at least 30 NDGD were required for a robust estimation of tensor orientation and mean diffusivity. Ni et al [11] found that $\mathrm{NDGD}=6$ and number of excitations (NEX) $=10$ were sufficient for estimation of FA values from region of interest (ROI) calculations. All these researchers mentioned above did their studies with the constraint of constant imaging time. Previous work by Poonawalla ${ }^{[12]}$ concluded that when the acquisition time was held constant, the sum of the diffusion tensor variances decreased as NDGD increased, and signal averaging may not be as effective as increasing NDGD, especially when NDGD is small (e.g., NDGD < 13).

In this paper, the SNR of fractional anisotropy (FA) maps, apparent diffusion constant mean (ADC-mean) maps, the largest eigenvalue $\lambda_{1}$ (LEV) maps, and eigenvector orientation (EVO) maps associated with $\lambda_{1}$ derived from diffusion tensor and the FA values calculated from ROI were compared in 7 protocols corresponding to different NDGD $(6,9,12,15,20,25$, and 30 noncollinear). Unlike in previous work where the NEX varied to keep imaging time constant, the number of images averaged $(\mathrm{NEX}=3)$ is fixed in this work so as to all the 7 protocols have the same original SNR. So the imaging time for the 7 protocols was not held constant and the higher NDGD protocols would be expected to perform better given that the imaging time was greater.

The purpose of this work is to independently determine the effect of NDGD on SNR of these tensor-derived measurement maps mentioned above with the fixed NEX.

\section{MATERIALS AND METHODS}

All DTI data used in this paper were downloaded from the website of Johns Hopkins Medical Institute Laboratory of Brain Anatomical MRI with permission.

\subsection{Subjects}

All images were acquired in eight healthy volunteers (three females, five males; range, 21-29 years). The subjects did not have any history of neurological diseases. Institutional review board approval was obtained for the study, and informed consent was obtained from all subjects.

\subsection{Data Acquisition}

A 1.5T MR scanner (Gyroscan NT; Philips Medical Systems, Best, the Netherlands) was used. DTI data were acquired by using a single-shot echo-planar imaging sequence with 7 protocols corresponding to different NDGD $(6,9,12,15,20,25$, and 30 noncollinear), and the $b$ value was $700 \mathrm{smm}^{-2}$. The image matrix was $256 \times$ 256 pixels, with a field of view of $246 \times 220 \mathrm{~mm}$ (nominal resolution, $2.2 \mathrm{~mm}$ ). Transverse sections of 2.2 $\mathrm{mm}$ thickness were acquired parallel to the anterior commissure-posterior commissure line. A total of 55 sections covered the entire hemisphere and brainstem without gaps. The acquisition time per dataset was approximately 6 minutes. All DW imaging were repeated 3 times, so they have the same original SNR. Five additional images for each slice with minimal DW (b_ $0=33$ $\mathrm{smm}^{-2}$ ) were also acquired, and all 7 DTI acquisitions have the same b_0 images.

\subsection{Definitions of DTI Measurements}

The ADC, which is used to characterize the water diffusion, can be calculated from the following Equation (1) [6].

$$
A D C_{k}=\frac{\ln \left(S_{k} / S_{0}\right)}{b},(\mathrm{k}=1,2 \ldots \mathrm{K} ; \mathrm{K} \geq 6)
$$

where, the constant $b$ is the diffusion-weighting factor, $\mathrm{S}_{0}$ is the signal obtained without diffusion gradient, and $\mathrm{S}_{\mathrm{k}}$ is the signals corresponding to the different gradient directions $(\mathrm{k}=1,2 \ldots \mathrm{K} ; \mathrm{K} \geq 6)$. ADC-mean can be calculated by averaging the set of $\mathrm{ADC}_{\mathrm{k}}$.

From the diffusion tensor, three eigenvalues, $\lambda_{1}>\lambda_{2}>\lambda_{3}$, which define the diffusion magnitude, can be determined by diagonalizing the tensor for each voxel. Three eigenvectors (associated with three eigenvalues), which describe the diffusivity in the three directions, can be calculated. Based on these three diffusivities, the FA commonly used for anisotropy definitions is calculated to yield values between 0 and 1 by the following Equation (2) [6].

$$
F A=\sqrt{\frac{3}{2}} \frac{\sqrt{\left(\lambda_{1}-\bar{D}\right)^{2}+\left(\lambda_{2}-\bar{D}\right)^{2}+\left(\lambda_{3}-\bar{D}\right)^{2}}}{\sqrt{\lambda_{1}{ }^{2}+\lambda_{2}{ }^{2}+\lambda_{3}{ }^{2}}}
$$

where,

$$
\bar{D}=\frac{\lambda_{1}+\lambda_{2}+\lambda_{3}}{3}
$$

\subsection{SNR Calculation}

SNR measures the roughness or granularity of diffusion tensor-derived measurement maps, it should be equal to the ratio of power spectrum of signal to that of noise. But in general, spectrum analyze is not recommended to estimate the SNR of magnetic resonance (MR) images because it is actually re-created from frequency-signal or $\mathrm{k}$-space. In addition, spectrum analyze is good for random signal (include random noise) analyze, this is not the case for the measurements derived from tensor calculation because the only resource of the random error during this tensor calculation comes from the finite bit length of the computers.

The method presented by Mouyan Zou [13] can be used to estimate approximately SNR of an image, which is the variance of signal divided by that of noise. According to the theory, local variance of all pixels of an image should be calculated, the maximum of the local variance which stands for the signal variance is divided by the minimum which stands for the noise variance, and the result (see Equation (3)) as the approximate SNR should be amended by empirical formula. 


$$
\mathrm{SNR}=\frac{\sigma_{\max }^{2}}{\sigma_{\min }{ }^{2}}
$$

where, $\sigma^{2}$ is an estimated value of the local variance.

Since "local variance" affects the SNR measurement, the local neighborhood included $10 \times 10$ pixels as an kernel was used to calculate the local variance in this study. The kernel of larger or smaller than $10 \times 10$ pixels was not suggested because the former resulted in lower SNR and the latter resulted in higher SNR.

The SNR calculated for the base images, which were averaged thrice, is the same for all 7 protocols $(\mathrm{SNR}=$ 67.85) by using this method.

\section{DATA PROCESSING}

The DW images were transferred to a workstation and processed by employing DTI Studio [6] developed for diffusion tensor images calculating and fiber tracking. For each DTI dataset, the six independent elements of the $3 \times 3$ diffusion tensor were calculated for each voxel. After diagonalization, three eigenvalues, $\lambda_{1}>\lambda_{2}>\lambda_{3}$, and three eigenvectors were calculated for each voxel, and in turn, LEV maps and EVO maps associated with $\lambda_{1}$ were obtained. Then FA maps and ADC-mean maps were also obtained by using Equations (2) and (1). EVO maps associated with $\lambda_{1}$ were used as an indicator of fiber orientation. On the EVO maps, red, green, and blue colors were assigned to right-left, anterior-posterior, and superior- inferior orientations, respectively [14].

Based on MatLab platform, estimating variance as an approach was used to provide a global estimate of SNR for these tensor-derived measurement maps that characterizes the uncertainty of the DTI measurements mentioned above in the 7 protocols corresponding to $6,9,12$, $15,20,25$ and 30 noncollinear NDGD with a 700 $\mathrm{mm}^{2} / \mathrm{sec} b$ value. To illustrate the effect of NDGD on FA values, we also calculated FA values within ROI (about 30 pixels) selected in the white matter from FA maps in

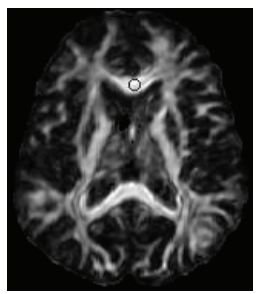

Figure 1. An FA map corresponding to 30 noncollinear diffusion gradient directions, with an ROI (about 30 pixels) in the white matter marked with a circle.
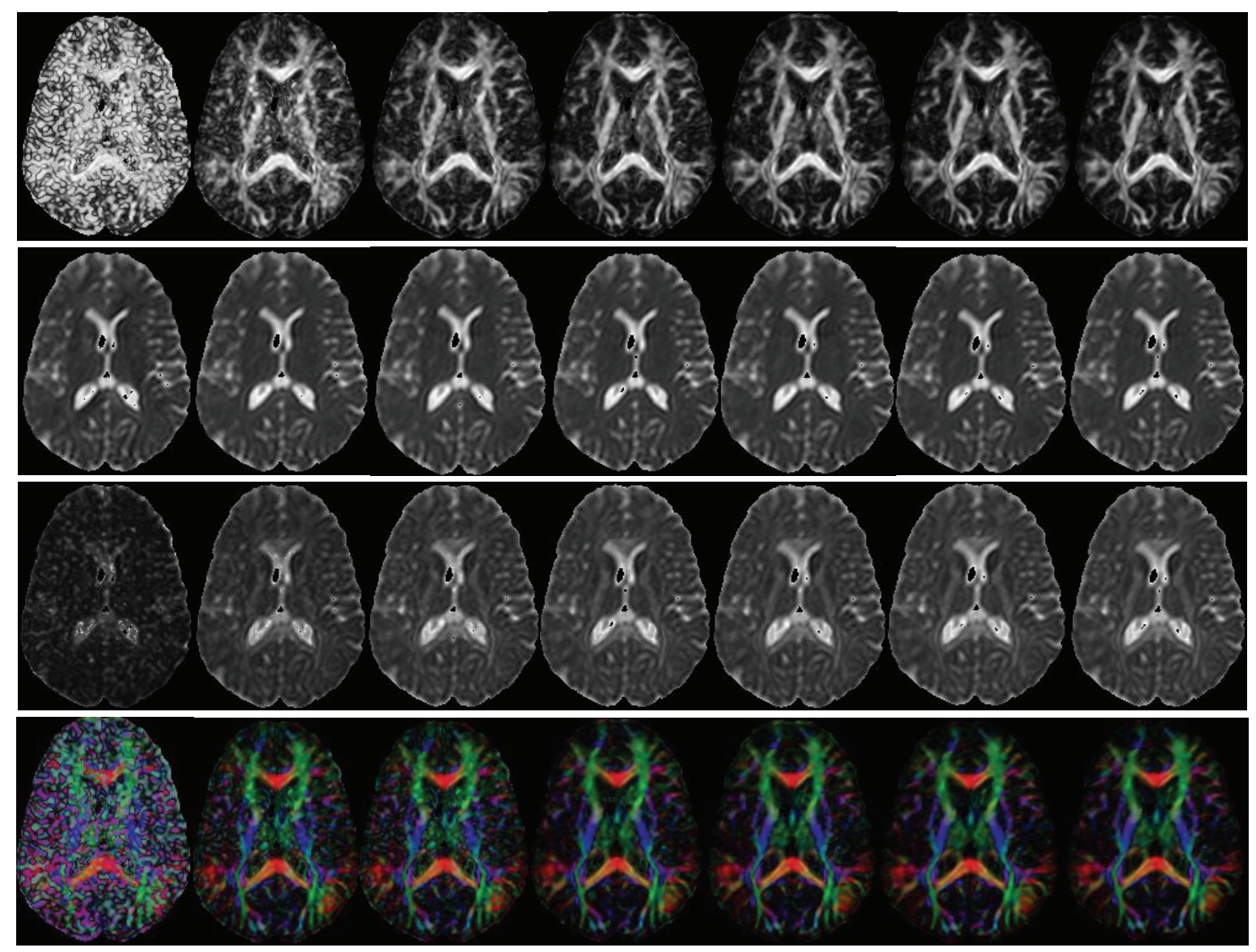

Figure 2. Tensor-derived measurement maps. The first, second, third, and forth rows are FA maps, ADC-mean maps, LEV maps, and color-coded maps for the eigenvectors associated with $\lambda 1$, respectively, which corresponding to $6,9,12,15,20,25$, and 30 noncollinear NDGD arranged from left to right. 
all 7 protocols. An FA map corresponding to 30 noncollinear NDGD with an ROI was shown in Figure 1.

An unpaired T-test for the SNR of these tensor-derived measurement maps and the FA values within ROI in the 7 protocols was performed by using SPSS11.5 software, a $P$ value less than 0.05 for a measurement was considered as statistically significant.

\section{RESULTS}

The tensor-derived measurement maps of one subject acquired from tensor calculation in the 7 protocols were shown in Figure 2. The improvement of the SNR with the NDGD increasing could be observed visually. The mean \pm standard deviation for the SNR of tensor-derived measurement maps and the FA values within ROI in the 7 protocols were listed in Table 1 . The SNR of these tensor-derived measurement maps varied with the NDGD increasing were shown in Figure 3. The correlations between FA values within ROI and NDGD were fitted by linear lines and shown in Figure 4.

In order to predigest the results, the $P$ values for the SNR of tensor-derived measurement maps and FA values within ROI in the 4 protocols corresponding to different NDGD $(6,12,20$, and 30 noncollinear) instead of all the 7 protocols were listed in Table 2.

From the curves in Figure 3, which demonstrate the SNR as a function of the NDGD for tensor-derived measurement maps, we note that the SNR of the tensor-derived measurement maps could be improved with more NDGD. Also it is obvious that the more NDGD were used, the higher SNR could be obtained from Fig- ure 2. This is perfectly accordant with the findings of D.K. Jones et al (i.e., when the NDGD is more than 6, which happens frequently in practice in order to improve the SNR and reduce the bias of tensor estimation) [15].

Both Figure 4 and all $P$ values $(>0.05)$ of FA values within ROI listed in Table 2 demonstrate that there are no significant variances in the FA values within ROI between any 2 protocols with the NDGD increasing, which are accordant with the conclusion of the previous original research by $\mathrm{Ni}$ et al [11].

\section{DISCUSSION}

Ni et al [11] found that NDGD $=6$ and NEX $=10$ were sufficient for estimation of FA from ROI calculations. But in this paper, the FA maps shown in the first column in Fig 2 look bad, it is considered that the NDGD is too low $(\mathrm{NDGD}=6)$, which results in low SNR for these tensor-derived measurement maps. Because our results were based on the same original SNR, i.e. all the DW images used in this study were averaged thrice, so the SNR of these tensor-derived measurement maps varied in the 7 protocols only is dependent on the NDGD.

The functional dependence of the SNR of each tensor-derived measurement on the NDGD is the most interesting result for this paper. The curves in Figure 3 and Figure 4 indicated that the SNR of FA maps, ADC-mean maps, LEV maps, and EVO maps associated with $\lambda_{1}$ were improved with the NDGD increasing, but there were no significant variances in the FA values within ROI among these 7 protocols (refer to the last column in Table 1).

Table 1. Mean \pm standard deviation for the SNR of tensor-derived measurement maps and the FA values within ROI in the 7 protocols. *NDGD represents number of diffusion gradient directions.

\begin{tabular}{cccccc}
\hline NDGD* & \multicolumn{2}{c}{ SNR of Tensor-derived Measurement } & \multicolumn{2}{c}{ FA values } \\
& FA Maps & ADC-mean & LEV Maps & EVO Maps & \\
\hline 6 & $35.78 \pm 1.39$ & $49.08 \pm 3.39$ & $34.39 \pm 1.20$ & $40.30 \pm 0.53$ & $0.79 \pm 0.06$ \\
9 & $51.65 \pm 1.43$ & $60.96 \pm 1.87$ & $39.96 \pm 1.54$ & $51.08 \pm 0.54$ & $0.73 \pm 0.08$ \\
12 & $61.62 \pm 2.24$ & $66.55 \pm 1.64$ & $44.09 \pm 1.73$ & $58.22 \pm 0.23$ & $0.75 \pm 0.08$ \\
15 & $63.98 \pm 1.67$ & $68.67 \pm 1.44$ & $45.95 \pm 1.38$ & $59.65 \pm 0.46$ & $0.76 \pm 0.08$ \\
20 & $69.39 \pm 1.66$ & $72.63 \pm 1.29$ & $48.22 \pm 1.57$ & $61.27 \pm 0.43$ & $0.74 \pm 0.09$ \\
25 & $71.15 \pm 2.46$ & $73.28 \pm 1.47$ & $53.16 \pm 1.26$ & $64.28 \pm 0.41$ & $0.74 \pm 0.08$ \\
30 & $72.45 \pm 7.52$ & $73.68 \pm 1.77$ & $57.44 \pm 1.65$ & $69.40 \pm 0.49$ & $0.74 \pm 0.08$ \\
\hline
\end{tabular}

Table 2. $\mathrm{P}$ values for the SNR of tensor-derived measurement maps and the FA values within ROI in the 4 protocols corresponding to different NDGD $(6,12,20$, and 30 noncollinear). *NDGD represents number of diffusion gradient directions.

\begin{tabular}{|c|c|c|c|c|c|}
\hline \multirow[t]{2}{*}{ NDGD* } & \multicolumn{5}{|c|}{$\mathrm{P}$ values for the SNR of tensor-derived measurement maps and the FA values within ROI } \\
\hline & FA values & ADC-mean Maps & LEV Maps & EVO Maps & FA values \\
\hline 6 vs 12 & $<<0.005$ & $<<0.005$ & 0.001 & $<<0.005$ & 0.287 \\
\hline 6 vs 20 & $<<0.005$ & $<<0.005$ & $<<0.005$ & $<<0.005$ & 0.177 \\
\hline 6 vs 30 & $<<0.005$ & $<<0.005$ & $<<0.005$ & $<<0.005$ & 0.150 \\
\hline 12 vs 20 & 0.010 & 0.011 & 0.046 & 0.050 & 0.725 \\
\hline 12 vs 30 & 0.011 & 0.009 & 0.001 & $<<0.005$ & 0.693 \\
\hline 20 vs 30 & 0.089 & 0.376 & 0.006 & 0.011 & 0.977 \\
\hline
\end{tabular}




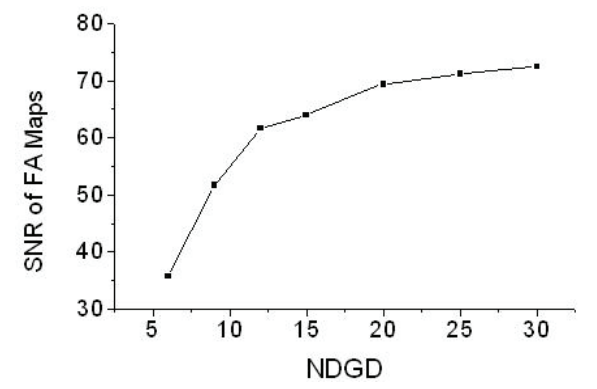

(a)

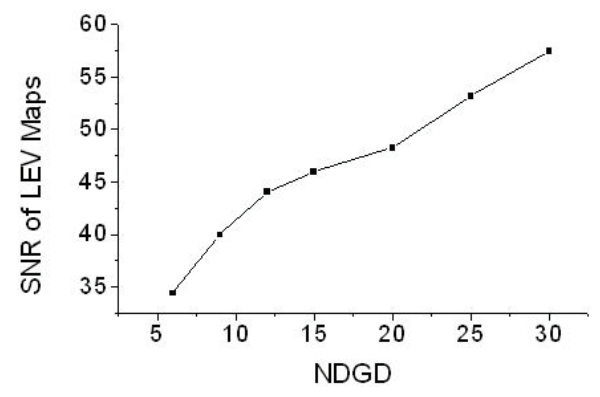

(c)

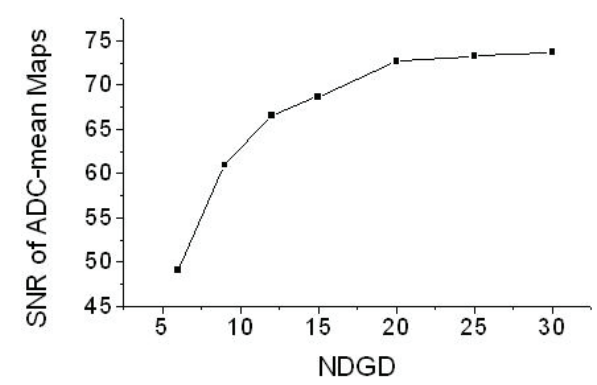

(b)

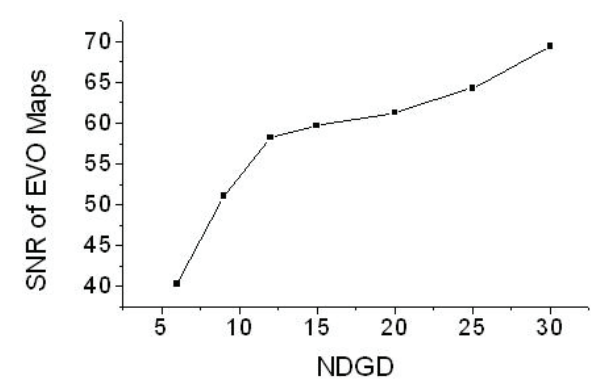

(d)

Figure 3. SNR of tensor-derived measurement maps vs NDGD.

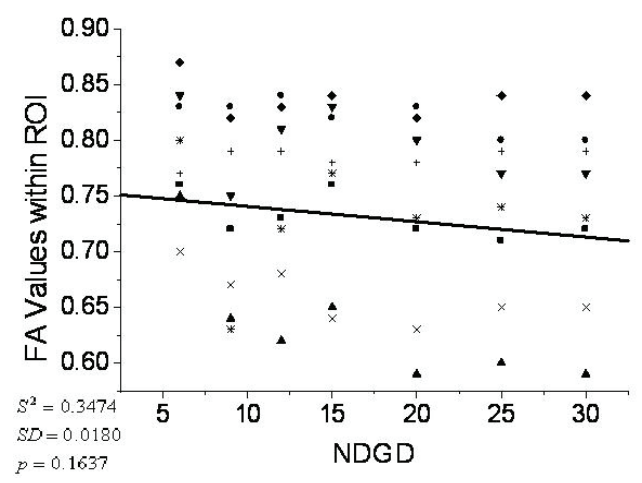

Figure 4. FA values within ROI vs NDGD.

In the previous studies, with the constant imaging time, some researchers investigated various protocols (different NDGD) in terms of the variance of FA measurements and demonstrated that a protocol employing 24 or 30 NDGD outperformed a protocol with only 6 NDGD [8, 9]. Papadakis NG et al considering three diffusion anisotropy maps concluded that the minimum NDGD required for robust anisotropy estimation was between 18 and 21[16].

In our study, we mainly focused on the effect of different NDGD on the SNR of tensor-derived measurement maps (FA maps, ADC-mean maps, LEV maps, and EVO maps associated with $\lambda_{1}$ ) in the 7 protocols with fixed NEX $=3$. The curves in Figure 3 (a) and (b) show that, for FA maps and ADC maps, there is a remarkable and linear improvement in the SNR when the NDGD increases from 6 to 12. Further improvement in the SNR (albeit less remarkable) is observed when the NDGD is further increased. Especially, there is no considerable difference occurred in the SNR when the NDGD increases from 20 to 30 , which means that it has little contribution to SNR of FA and ADC-mean maps when NDGD is more than 20. $P$ values $(20$ vs 30$)=0.089$ for FA maps and 0.376 for ADC maps in the Table 2 are much more than 0.05 , which also demonstrates that there is no significant improvement in SNR by increasing the NDGD from 20 to 30 . This is consistent with the conclusion suggesting that 20-diffusion gradients be probably sufficient for in vivo human study of diffusion anisotropy [10].

For LEV maps and EVO maps associated with $\lambda_{1}$, seen from the curve in Figure 3 (c) and (d), there is a significant improvement in SNR by increasing the NDGD from 6 to 12 and from 20 to 30. The curve increases almost linearly when NDGD increases from 6 to 30 for SNR of LEV maps. $P$ values $(20$ vs 30$)=0.006$ for LEV maps and 0.011 for EVO maps associated with $\lambda_{1}$ in the Table 2, which are less than 0.05, also demonstrate that the SNR of LEV maps and EVO maps associated with $\lambda_{1}$ are significant different between 20 and 30 diffusion gradients. This suggests that the NDGD less than 30 be not enough for tensor-orientation estimation, which also is consistent with the results from Monte Carlo study [10].

Therefore, the more NDGD are used, the higher SNR would be obtained. But in clinical applications of DTI, the total scanning time could not be too long because of the artifacts caused by patients' motion. So an optimum NDGD for DTI data acquisition is needed due to both the requirements and limitations mentioned above. There would be a trade-off between the NDGD and clinical limitations. 


\section{CONCLUSION}

7 different types of results derived from 6, 9, 12, 15, 20, 25 , and 30 noncollinear NDGD, respectively, have been compared in terms of SNR of tensor-derived measurement maps and FA values within ROI based on Matlab platform. The SNR of FA maps and ADC-mean maps increased linearly as the NDGD increased from 6 to 20 . And the curves were almost level as the NDGD increasing from 20 to 30. For SNR of LEV and EVO maps, the curves were linearly direct ratio to the NDGD. FA values within ROI were independent of NDGD. This study provides insight into the effect of NDGD on SNR and may be useful in understanding the tradeoffs involved in DTI acquisition design.

\section{ACKNOWLEDGMENTS}

We are grateful to Dr. Hangyi Jiang who works in Johns Hopkins University School of Medicine, Dr. Maolin Qiu who works in Yale University, and Dr. Bob L. Hou from Memorial Sloan Kettering Cancer Center for their helpful discussions and encouraging comments during the course of this study. In addition, we thank Dr. Hangyi Jiang again for supplying the data and the software (DTI Studio) for our study. Finally, we would like to thank the reviewers for their valuable remarks.

\section{REFERENCES}

[1] M. Jackowski, C. Y. Kao, M. L. Qiu, et al. (2005) White matter tractography by anisotropic wavefront evolution and diffusion tensor imaging. Medical Image Analysis, 9, 427-440.

[2] T. McGraw, B.C. Vemuri, Y. Chen, et al. (2004) DT-MRI denoising and neuronal fiber tracking. Medical Image Analysis, 8, 95-111.

[3] D. L. Bihan, J. F. Mangin, C. Poupon, et al. (2001) Diffusion Tensor Imaging: Concepts and Applications. Journal of Magnetic Resonance Imaging, 13, 534-546.
[4] P. J. Basser, J. Mattiello, D. LeBihan. (1994) Estimation of the effective selfdiffusion tensor from theNMRspin echo. JMagn Reson B, 103, 247-254.

[5] P. J. Basser, J. Mattiello, D. LeBihan. (1994) MR diffusion tensor spectroscopy and imaging. Biophys J, 66, 259-267.

[6] H. Jiang, P. C.M. van Zijl, et al. (2006) DtiStudio: Resource program for diffusion tensor computation and fiber bundle tracking. computer methods and programs in biomedicine, 8 1,106-116.

[7] D. S. Tuch. (2004) Q-Ball Imaging. Magnetic Resonance in Medicine, 52, 1358 - 1372

[8] N. G. Papadakis, D. Xing, G. C. Houston, et al. (1999) A study of rotational invariant and symmetric indices of diffusion anisotropy. Magn Reson Imaging, 17, 881-92.

[9] S. Skare, M. Hedehus, M.E. Moseley, et al. (2000) Condition number as a measure of noise performance of diffusion tensor data acquisition schemes with MRI. J Magn Reson, 147, 340-52.

[10] D. K. Jones. (2004) The effect of gradient sampling schemes on measures derived from diffusion tensor MRI: a Monte Carlo study. Magn Reson Med, 51, 807-15.

[11] H.Ni, V.Kavcic. T. Zhu, et al. (2006) Effects of Number of Diffusion Gradient Directions on Derived Diffusion Tensor Imaging Indices in Human Brain. AJNR Am J Neuroradiol, 27,1776-81

[12] A. H. Poonawalla, MS, X. H Joe Zhou, PhD *. (2004) Analytical error propagation in diffusion anisotropy calculations. JMRI, 19, 489-498

[13] M. Zou (2001) Deconvolution and Signal recovery. Publishing Company of National Defence and Industry (Chinese book).

[14] S Pajevic, C Pierpaoli. (1999) Color schemes to represent the orientation of anisotropic tissues from diffusion tensor data: application to white matter fiber tract mapping in the human brain. Magn Reson Med, 42, 526-540.

[15] D.K. Jones, M.A. Horsfield. (1999) A. Simmons. Optimal strategies for measuring diffusion in anisotropic systems by magnetic resonance imaging. Magn. Reson. Med, 42 (3), 515-525.

[16] N.G. Papadakis, C. D. Murrills, L. D. Hall, et al. (2000) Minimal gradient encoding for robust estimation of diffusion anisotropy. Magn Reson Imaging, 18, 671-679. 